\title{
Airborne Polarimetric Remote Sensing for Atmospheric Correction
}

\author{
Tianquan Liang, Xiaobing Sun, Han Wang, Rufang Ti, and Cunming Shu \\ Key Laboratory of Optical Calibration and Characterization, Anhui Institute of Optics and Fine Mechanics, \\ Chinese Academy of Science, Hefei 230031, China \\ Correspondence should be addressed to Tianquan Liang; liangpolaris@126.com
}

Received 2 October 2014; Revised 9 January 2015; Accepted 17 January 2015

Academic Editor: Seong G. Kong

Copyright (C) 2016 Tianquan Liang et al. This is an open access article distributed under the Creative Commons Attribution License, which permits unrestricted use, distribution, and reproduction in any medium, provided the original work is properly cited.

The problem, whose targets can not be effectively identified for airborne remote sensing images, is mainly due to the atmospheric scattering effect. This problem is necessary to be overcome. According to the statistical evaluations method and the different characteristics of polarization between the objects radiance and atmospheric path radiation, a new atmospheric correction method for airborne remote sensing images was proposed. Using this new method on the airborne remote sensing images which acquired on the north coast areas of China during the haze weather, we achieved a high quality corrected atmosphere-free image. The results demonstrate the power of the method on the harbor area. The results show that the algorithm, improving image contrast and image information entropy, can effectively identify the targets after atmospheric correction. The image information entropy was enhanced from 5.59 to 6.62. The research provides a new and effective atmospheric correction technical approach for the airborne remote sensing images.

\section{Introduction}

Airborne remote sensing plays an important role in searching materials of interest [1], as well as an important platform of national science missions for remote sensing monitoring. This technique exhibits many potentialities in a large amount of applications such as geology mapping, land resources planning, terrain cover information classification, monitoring growth situation of crop, ocean targets detecting, and military surveillance. It is especially important that the aerial remote sensing can provide real-time, actionable data, automatic detection, and classification. The data acquired by aerial remote sensing platform consists of objects radiance and atmospheric path radiance information. The scene is strongly affected by the presence of the atmosphere along the path from the sun to the target to the sensor. These potentialities have motivated the development of atmospheric correction [2].

One of the simplest methods of atmospheric correction is the dark object subtraction method $[3,4]$. This method approximates the path radiance from the darkest object in the scene. Currently, different methods are used to solve the direct transfer radiative problems; for example, they provide atmospheric parameters for atmospheric correction, such as 6S (Second Simulation of the Satellite Signal in the Solar Spectrum) model $[5,6]$. It provides a computer code which can accurately simulate atmospheric radiative. There are some other standard procedures such as LOWTRAN and MODTRAN $[7,8]$. In the atmospheric transfer equation, it can provide powerful atmospheric parameters to recover the scenes [9]. The atmospheric correction has been experienced for long history. There have been several types of approaches: scene-based empirical approaches, radiative transfer modeling approaches, and hybrid approaches [10, 11].

Recently some researchers have used as few as two images taken through a polarizer at different orientations in the haze weather to recover the scene based on effective atmospheric degradation physical model $[12,13]$. The algorithm gives birth to better results, but the work concentrated on the horizontal direction and the scene should include the sky region to estimate the unknown parameters. Christoph Borel accomplished the atmospheric correction by using the recently 
released vectorized version of $6 \mathrm{~Sv}$ of airborne POLDER polarimetric imagery. However, the approach has limitations and the typical bilinear, thin plates spline, and cubic 2-D interpolation methods produced unsatisfactory results [14]. We take advantage of polarization information and statistical evaluations information for the airborne polarization image atmospheric correction. The basic statistical shows that it is very often that some pixels have very low intensity. The pixels were completely shadowed in some case and their radiances were mainly contributed by atmospheric scattering (called "path radiance"). The main advantages of statistical evaluations algorithm are that it is effective and easy to estimate the parameters. These parameters are necessary for the airborne image correction. Compared to other atmospheric correction methods, it does not require any real time ground measurement parameters so that it is simple and relatively straightforward to apply. We construct the airborne polarization remote sensing atmospheric correction model which combined polarization information and statistical evaluations method. The image contrast and entropy are increased dramatically when this algorithm is applied in the airborne polarization remote sensing image acquired in the haze weather.

\section{Principles and Method}

To make the paper self-contained, this section will review the known formation model of haze images, as shown in Figure 1.

The airborne polarization remote sensing acquired images composed of two main components. The first originated from the object radiance. Let us denote by $J(x, y)$ the object radiance as if it was taken in clear atmospheric circumstances, without scattering and absorbing in the line of sight. Due to attenuation in the haze, the airborne polarization camera senses a fraction of this radiance. This attenuated signal is called direct transmission. The second component is known as path radiance, or airlight, or ambient light. It originates from the atmosphere illumination (e.g., sunlight), a particle radiates the light it absorbs, behaving as a light source suspending in the atmosphere, the light scattered a great many times by a huge number of particles. All these particles generate the atmospheric light, a portion of which is scattered into the line of sight by the atmospheric. The model can be described as follows:

$$
I(x, y)=J(x, y) t(x, y)+A(1-t(x, y))
$$

where $I(x, y)$ is the total intensity which acquired by airborne polarization sensor and $t(x, y)$ is the medium transmission which describing the portion of the light that is not scattered and reaches the airborne polarization sensor. The goal of atmospheric correction is to recover $J(x, y)$ from $I(x, y)$. $t(x, y)=\exp (-\beta(\lambda) * z(x, y))$ called atmospheric transmission impact factor, where $\beta(\lambda)$ represents the atmospheric extinction coefficient which is determined by the particle material, size, shape, and concentration. $z(x, y)$ indicates the airborne polarization sensor flying height information. "-" indicates the light is weakened.

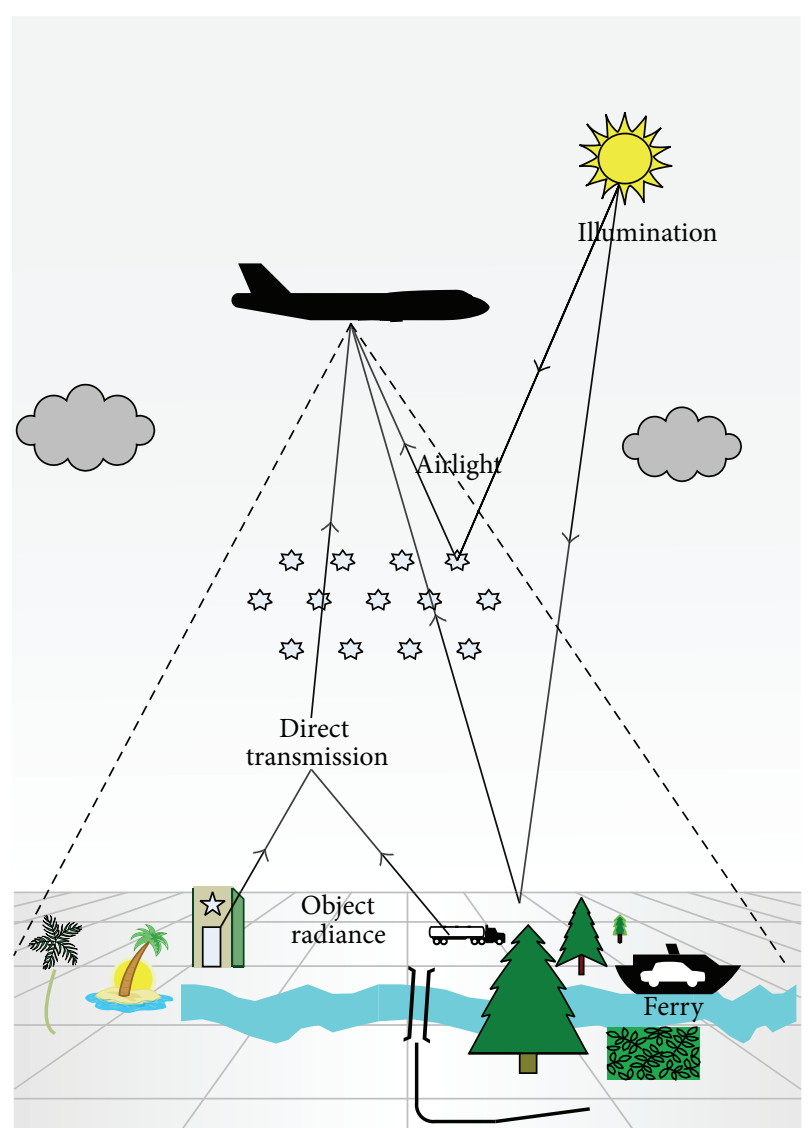

Figure 1: The image model for airborne polarization remote sensing.

We will observe strong polarization effect of atmospheric path radiance due to scattering by the atmosphere particles. The assumption which we make in this paper is that light emanating from objects has insignificant polarization. Because scattering does not change the direct transmission light polarization state, it follows that the polarization of the direct transmission is also insignificant. Thus the polarization of the atmospheric path radiance dominates the airborne polarization sensor measured light. As a result atmospheric path radiance information can be expressed as

$$
\begin{aligned}
A(1-t(x, y)) & =A^{\mathrm{atm}}(x, y)_{\max }+A^{\mathrm{atm}}(x, y)_{\min } \\
& =\frac{A^{\mathrm{atm}}(x, y)_{\max }-A^{\mathrm{atm}}(x, y)_{\min }}{p_{A}} \\
& \approx \frac{I(x, y)_{\max }-I(x, y)_{\min }}{p_{A}},
\end{aligned}
$$

where $p$ is atmospheric path radiance degree of polarization. It can be defined as

$$
p_{A}=\frac{A^{\mathrm{atm}}(x, y)_{\max }-A^{\mathrm{atm}}(x, y)_{\min }}{A^{\mathrm{atm}}(x, y)_{\max }+A^{\mathrm{atm}}(x, y)_{\min }} .
$$




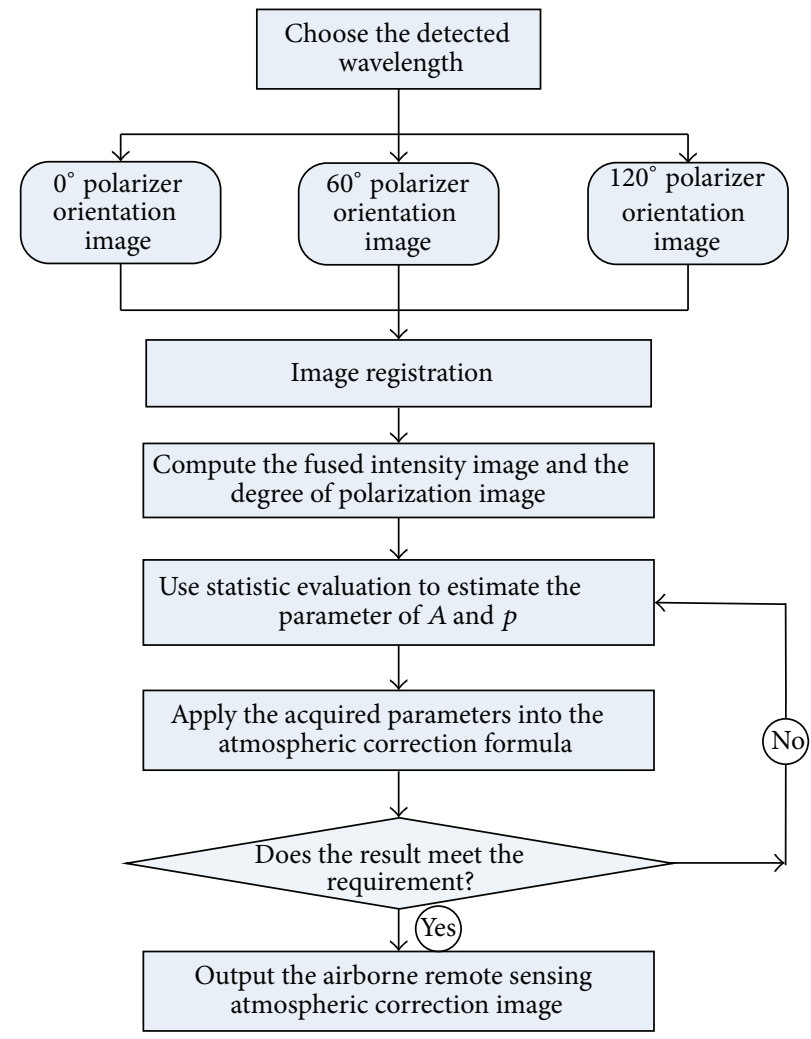

FIGURE 2: The flowchart of the airborne polarized atmospheric correction method.

According to above analysis, the path radiance can be derived from (2) and (3). The atmospheric transmission impact factor $t(x, y)$ can be expressed as (5). Consider

$$
\begin{gathered}
A^{\mathrm{atm}}=\frac{I(x, y) \times P(x, y)}{p_{A}(x, y)} \\
t(x, y)=1-\frac{I(x, y) \times P(x, y)}{A(x, y) \times p_{A}(x, y)} .
\end{gathered}
$$

Notice that $A$ represents the atmospheric path radiance without influence of object radiance. The parameters $A$ and $p_{A}$ were unknown. How do we estimate these parameters? We will bring forth new ideas to give the value of these parameters. It is a innovate method distinguish from other methods. It is statistical evaluations method, and we use statistic for all the pixels and then set threshold value to estimate these parameters, because we find that the images acquired by the airborne polarization sensor will have dark pixels which have low intensities. It is mainly due to some factors, like shadows effect and dark objects or surfaces, for example. We will explicitly introduce the computation procedure at the end of this section.

Polarization property of electromagnetic radiation can be described by the Stokes vector $(I, Q, U, V)^{T}$ [15]. Because the natural objects have low value of circular polarization, in practical measurement we do not consider circular polarization component, namely, the $V$ component of the Stokes vector. Utilizing a single rotatable linear polarizer, we set the reference direction, and the Stokes vector can be calculated by rotating three arbitrary polarization orientation angles of polarizer:

$$
I(\alpha)=\frac{1}{2}(I+Q \cos 2 \alpha+U \sin 2 \alpha),
$$

where $\alpha$ is the angle between the reference direction axis and the orientated direction. Stokes vector can deduce the degree of polarization:

$$
P=\frac{\sqrt{Q^{2}+U^{2}}}{I} .
$$

Based on these discussions, the final airborne atmospheric correction result, namely, the objects radiance $J(x, y)$, is recovered by

$$
J(x, y)=\frac{I(x, y)-\left(I(x, y) \times P(x, y) / p_{A}(x, y)\right)}{1-\left(I(x, y) \times P(x, y) / A(x, y) \times p_{A}(x, y)\right)} .
$$

In order to give an intuitive act of the airborne polarized atmospheric correction method, it is necessary to depict the flowchart of correcting method as shown in Figure 2.

\section{Instrument and Experiment Description}

We use the instrument named the Directional Polarimetric Camera (DPC) which was designed by Anhui Institute of Optics and Fine Mechanics, Chinese Academy of 


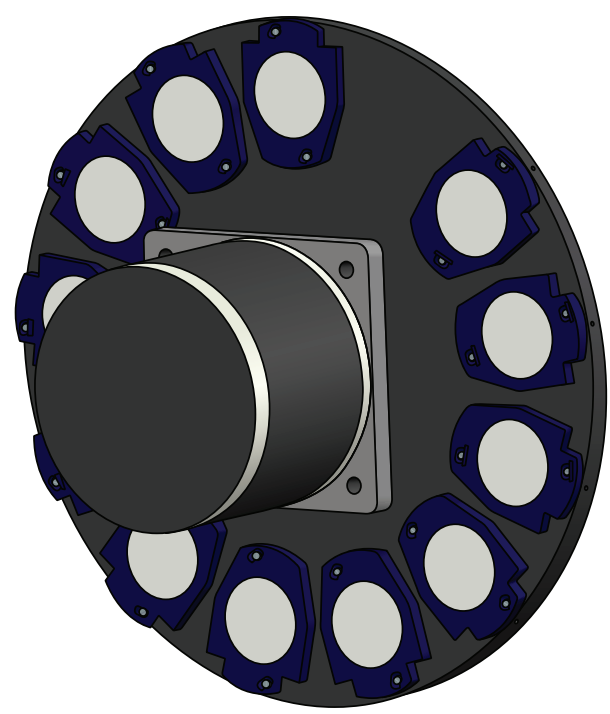

Figure 3: Polarizer/filter wheel.

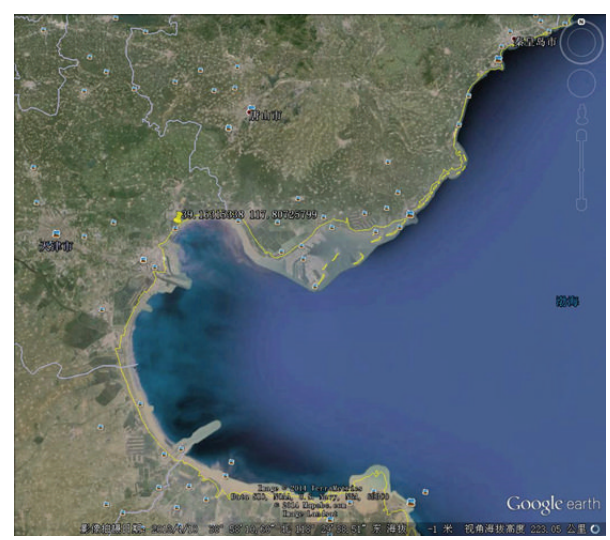

Figure 4: Tianjin-Bohai-Tangshan areas in China’s north coast.

Science [16]. It has three polarized wavebands $(495 \mathrm{~nm} /$ $670 \mathrm{~nm} / 870 \mathrm{~nm}$ ) and three unpolarized wavebands $(555 \mathrm{~nm} /$ $780 \mathrm{~nm} / 815 \mathrm{~nm}$ ). It recorded images through a rotated filter wheel with spectral filter and in three polarized spectral bands with linear polarized filters orientated at $0^{\circ}, 60^{\circ}$, and $120^{\circ}$. The images needed to be registered before spectra or the Stokes vectors can be computed. The wheel was shown in Figure 3. It is divided into thirteen sections. The FOV is from $-30^{\circ} \sim+30^{\circ}$ and on the diagonal it is from $-40^{\circ} \sim+40^{\circ}$.

The DPC have taken three flights experiments at TianjinBohai-Tangshan areas of China north coast (as shown on Figure 4) during March 21/26/27, 2012. The experiment data were huge; one flight acquired almost twenty thousand images. The scenes included farmland, city circle, countryside, harbor, and ocean. The weather was haze. As seen from the airplane the scenes were obscured by the atmosphere.

The plane has fixed a POS instrument with DPC at the same time. It will record the plane's position information including latitude, longitude, and altitude. It can create highly accurate flight mission planning and accurate sensor positioning and image acquisition. According to the POS

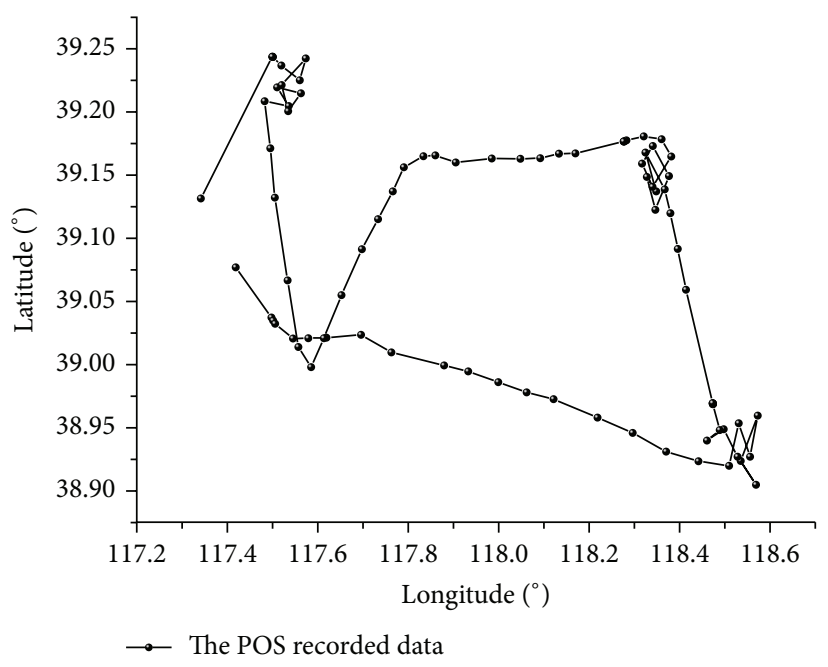

FIgure 5: The accurate flying trace in the Tianjin-Bohai-Tangshan area.

recorded information, we depicted the accurate flying trace of Tianjin-Bohai-Tangshan area as shown in Figure 5. The flight mission sets three important observer areas. The flying routes were intensive in the interesting areas as shown in Figure 5.

\section{Experiment Results}

The experiment data which we use were taken on March 21,2012 . We choose one group $670 \mathrm{~nm}$ data to prove our method. The data which we choose were acquired at coast $\left(39^{\circ} 09^{\prime} 19.78^{\prime \prime} \mathrm{N}, 117^{\circ} 48^{\prime} 29.83^{\prime \prime} \mathrm{E}\right)$; the flying altitude was $3608 \mathrm{~m}$. Because the DPC structure adopts polarizer/spectral filter wheel, one group data will have some difference scenes. Generating images that need to be registered before the Stokes vectors can be computed. This procedure was depicted in the flowchart as shown in Figure 2.

In this section we first display the original images which DPC acquired in the haze weather. It includes three different polarizer orientation angles (namely, $0^{\circ}, 60^{\circ}$, and $120^{\circ}$ ), as shown in Figure 6.

We then use the airborne polarization atmospheric correction method to recover the scene. The scene which we chose was a port at Tianjin, China. This port changed into a park, because of "Kyiv" aircraft carrier which China government bought from Russia. Our approach requires image registration for three different polarizer orientation angle images, because of the plane is flying when record the scene it will have some difference in sequence images. Using the registered images we can compute the fused intensity image and the degree of the polarization image. Then we use statistical evaluations to estimate the unknown parameters $A$ and $p_{A}$. Finally, we apply the acquired parameter to recover the scene, namely, the airborne polarization image atmospheric correction. The atmospheric correction results are as shown in Figure 7. The contrast of features in the atmospheric corrected image is greatly improved relative to the fused intensity image and three original polarizer orientation 


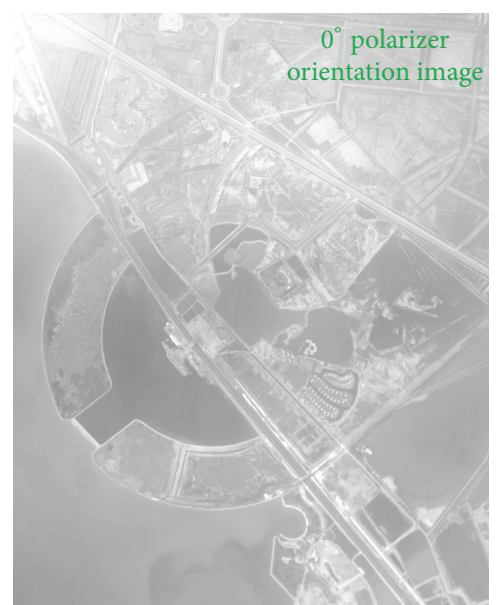

(a)

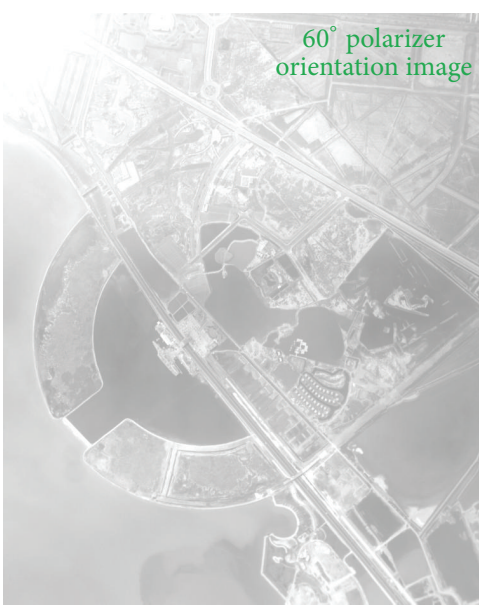

(b)

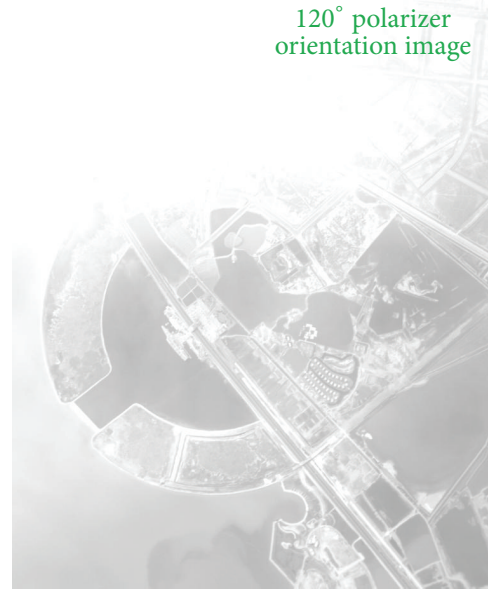

(c)

FIGURE 6: The raw images of three different polarizer orientation angles $\left((\mathrm{a}) /(\mathrm{b}) /(\mathrm{c})\right.$ represented $0^{\circ} / 60^{\circ} / 120^{\circ}$ polarizer orientation angle images, resp.).

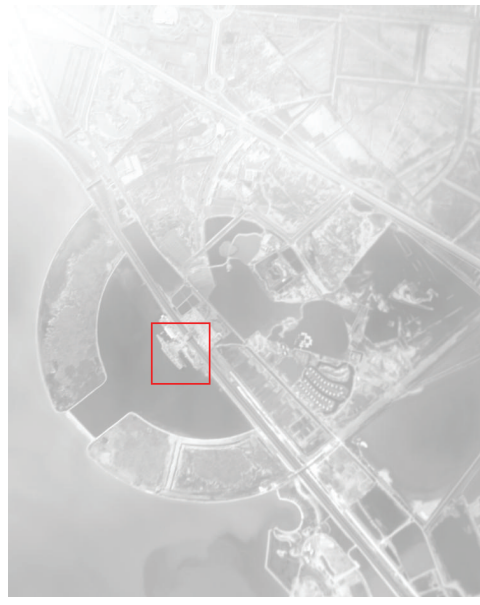

(a)

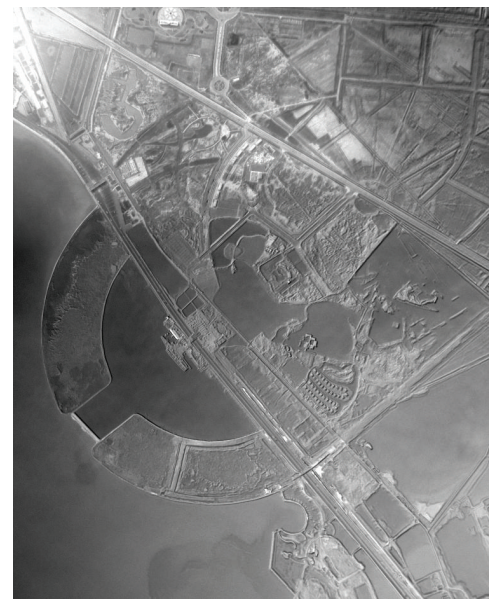

(b)

Figure 7: The fused intensity image (a) and the atmospheric corrected image (b).

angles raw images. Moreover, the algorithm removed the glint light, which existed in the raw images. Thus the "Kyiv" aircraft carrier is clearly visible in the scene, while in the raw images that area looks like white and blur. The recovered image show details unseen in the raw images of Figure 7.

In order to give restricted and objective results, atmospheric corrected results should make quantitative evaluations. We chose aircraft carrier area as shown in Figure 8, the red rectangle area marked in Figure 7. Compare the histogram of atmospheric correction results as shown in Figure 9. After atmospheric correction the image nonzero value distributed broadly and evenly. It proves that the image contrast is higher than the intensity image. We chose the aircraft carrier as the target and computed the average value of the target area. We chose the ocean around the target as the background and computed the average value of the background area. The object and background area were chosen as the same area of intensity image and atmospheric corrected image, as shown in Figure 8. The image contrast was enhanced from 0.058 to 0.536 . The contrast principle was defined as

$$
R=\frac{\left|f_{\text {object }}-f_{\text {background }}\right|}{f_{\text {background }}} .
$$

In 1948, Shannon introduced a general uncertainty measure on random variables which takes different probabilities among states into account [17]. Today this measure is known as "Shannon's entropy." Given events occurring with probability $P$, the Shannon entropy is defined as

$$
H=\sum_{i=1}^{m} P_{i} \log _{2} \frac{1}{P_{i}}=-\sum_{i=1}^{m} P_{i} \log _{2} P_{i}
$$

Shannon's entropy can compute an image, where probabilities of the gray level distributions are considered in the 


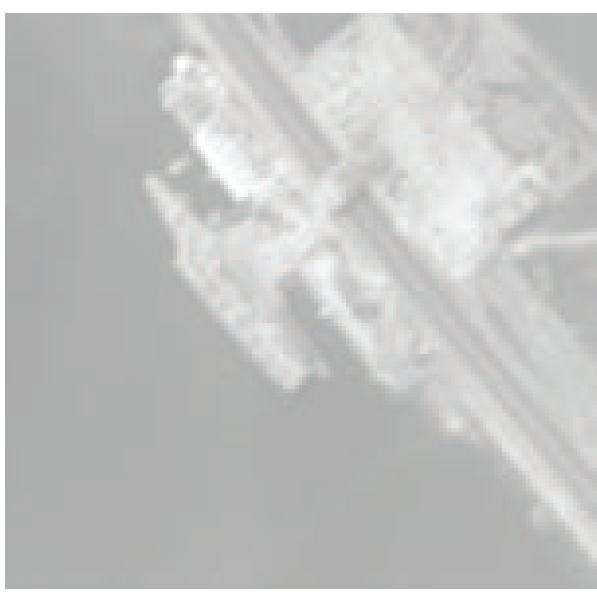

(a)

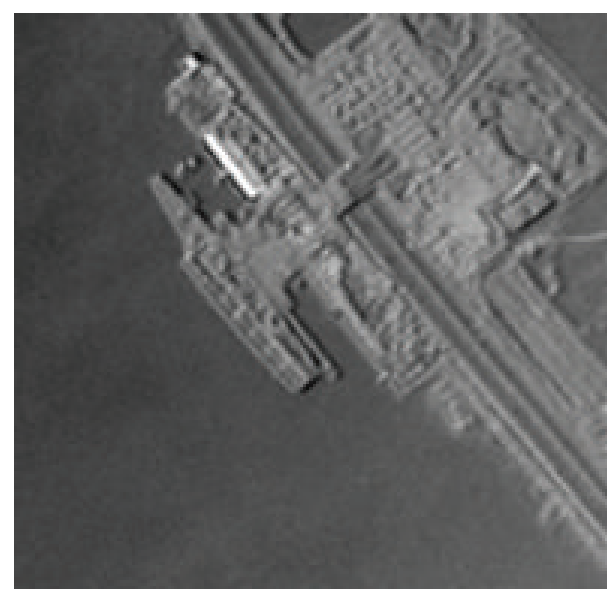

(b)

FIGURE 8: The red rectangle area marked in Figure 7 before (a) and after (b) atmospheric correction.

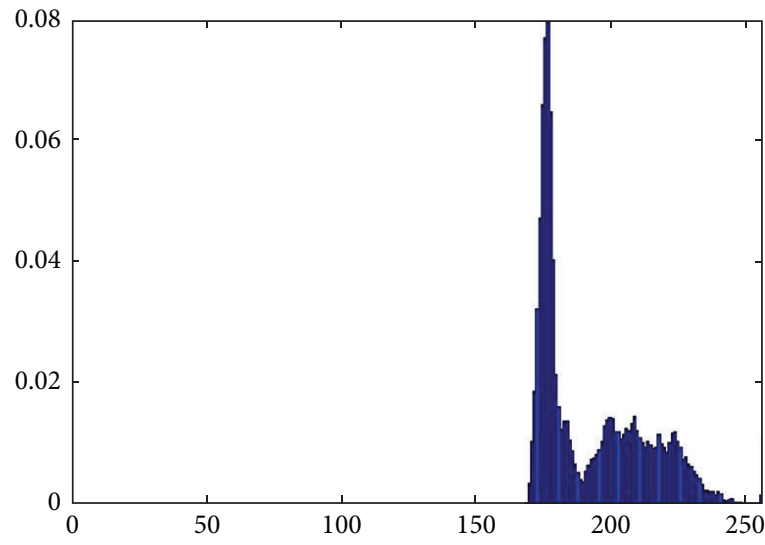

(a)

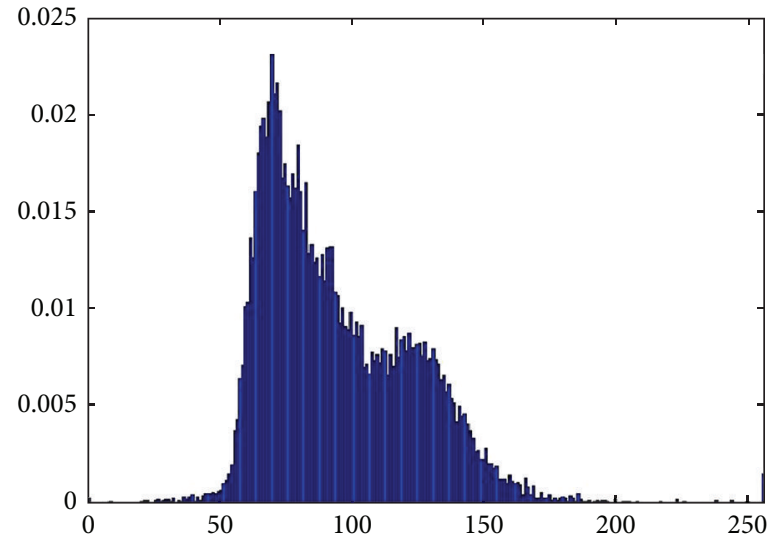

(b)

FIGURE 9: The normalized histogram of before (a) and after (b) atmospheric correction.

Shannon entropy formula. A probability distribution of gray values can be estimated by counting the number of times each gray value occurs in the image and dividing those numbers by the total number of occurrences. The image scene is more disorderly (namely, the scene objects more clearly), and the Shannon entropy is higher. After atmospheric correction, the entropy of intensity image has been enhanced from 5.59 to 6.62. As a result of the effect of atmosphere on the intensity image, which has led the whole system relatively orderly, the scenes texture is blurring. The whole system of atmospheric corrected image is more complex and the scenes details texture become clear.

\section{Conclusions}

In this paper, an approach for airborne polarization atmospheric correction was presented. It is based on analysis of images acquired through Directional Polarimetric Camera (DPC). The method is physics-based which enables a very effective recovery of the scene. It is a potentially useful tool for airborne atmospheric correction and target recognition applications. Additional work certainly needs to be done to further reach the goal of making the method full proof. In addition, further research and development of hardware are needed to obtain fast acquisition that does not need image registration and can handle uneven atmosphere.

\section{Conflict of Interests}

The authors declare no conflict of interests.

\section{Acknowledgments}

The authors would like to thank Professor Seong Kong and anonymous reviewer(s) for their valuable comments and suggestions to improve this paper. This work was supported by the National Basic Research Program (973) of China (no. 2010CB950803, no. 61322502). 


\section{References}

[1] C. Archer and J. Morgenstern, "Improved target recognition with live atmospheric correction," in Algorithms and Technologies for Multispectral, Hyperspectral, and Ultraspectral Imagery XIX, vol. 8743 of Proceedings of the SPIE, Baltimore, Md, USA, May 2013.

[2] S. Liang, Quantitative Remote Sensing of Land Surfaces, Science Press, 2009.

[3] J. Chavea, "Image-based atmospheric corrections-revisited and improved," Photogrammetric Engineering \& Remote Sensing, vol. 62, no. 9, pp. 1025-1036, 1996.

[4] M. S. Moran, R. D. Jackson, P. N. Slater, and P. M. Teillet, "Evaluation of simplified procedures for retrieval of land surface reflectance factors from satellite sensor output," Remote Sensing of Environment, vol. 41, no. 2-3, pp. 169-184, 1992.

[5] E. F. Vermote, D. Tanré, J. L. Deuzé, M. Herman, and J.-J. Morcrette, "Second simulation of the satellite signal in the solar spectrum, 6s: an overview," IEEE Transactions on Geoscience and Remote Sensing, vol. 35, no. 3, pp. 675-686, 1997.

[6] E. F. Vermote, D. Tanre, J. L. Deuze et al., Second Simulation of the Satellite Signal in the Solar Spectrum (6S), 6S User Guide Version 6.0, NASA-GSFC, Greenbelt, Md, USA, 1994.

[7] J. M. van den Bosch and R. E. Alley, "Application of LOWTRAN 7 as an atmospheric correction to airborne visible/infrared imaging spectrometer (AVIRIS) data," in Proceedings of the 10th Annual International Geoscience and Remote Sensing Symposium (IGARSS '90), pp. 175-177, May 1990.

[8] M. W. Matthew, S. M. Adler-Golden, A. Berk et al., "Status of atmospheric correction using a MODTRAN4-based algorithm," in Algorithms for Multispectral, Hyperspectral, and Ultraspectral Imagery VI, vol. 4049 of Proceedings of the SPIE, pp. 199-207, April 2000.

[9] X. Xiru, The Physics of Remote Sensing, Peking University Press, 2006.

[10] S. Liang and H. Fang, "An improved atmospheric correction algorithm for hyperspectral remotely sensed imagery," IEEE Geoscience and Remote Sensing Letters, vol. 1, no. 2, pp. 112-117, 2004.

[11] Y. J. Kaufman and C. Sendra, "Algorithm for automatic atmospheric corrections to visible and near-IR satellite imagery," International Journal of Remote Sensing, vol. 9, no. 8, pp. 13571381, 1988.

[12] Y. Y. Schechner, S. G. Narasimhan, and S. K. Nayar, "Polarization-based vision through haze," Applied Optics, vol. 42, no. 3, pp. 511-525, 2003.

[13] Y. Y. Schechner, S. G. Narasimhan, and S. K. Nayar, "Instant dehazing of images using polarization," in Proceedings of the IEEE Computer Society Conference on Computer Vision and Pattern Recognition (CVPR '01), vol. 1, pp. 325-332, 2001.

[14] C. Borel and C. Spencer, "Atmospheric correction of airborne POLDER polarimetric imagery using vectorized 6S," in Imaging Spectrometry XI, vol. 6302 of Proceedings of SPIE, pp. 63020T-163020T-12, August 2006.

[15] J. R. Schott, Fundamentals of Polarimetric Remote Sensing, SPIE Press, 2009.

[16] C. Ligang, Study of laboratory calibration of the airborne polarization CCD camera with wide field of view [Ph.D. thesis], Graduate University of Chinese Academy of Sciences, 2008.
[17] J.-B. Jeon, J.-H. Kim, J.-H. Yoon, and K.-S. Hong, "Performance evaluation of teeth image recognition system based on difference image entropy," in Proceedings of the 3rd International Conference on Convergence and Hybrid Information Technology (ICCIT '08), pp. 967-972, November 2008. 


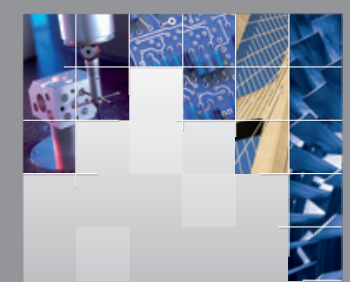

\section{Enfincering}
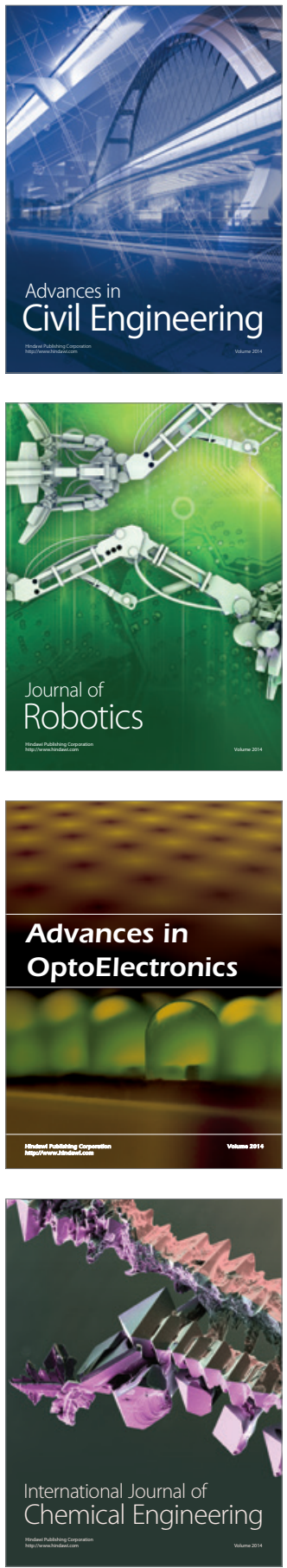

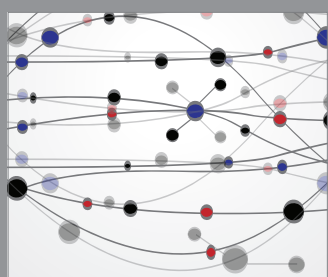

The Scientific World Journal

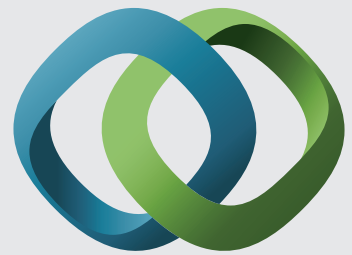

\section{Hindawi}

Submit your manuscripts at

http://www.hindawi.com
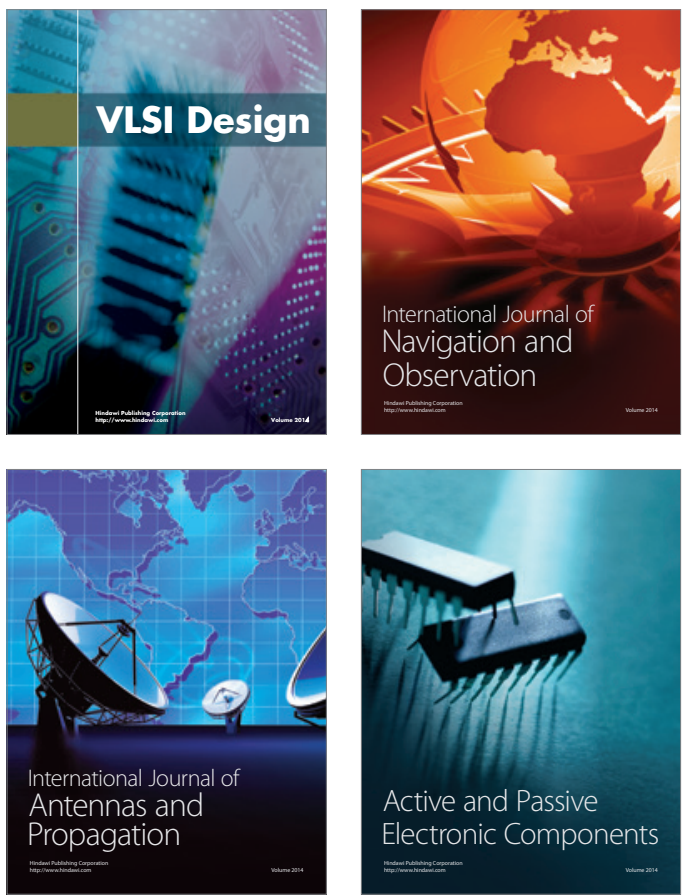
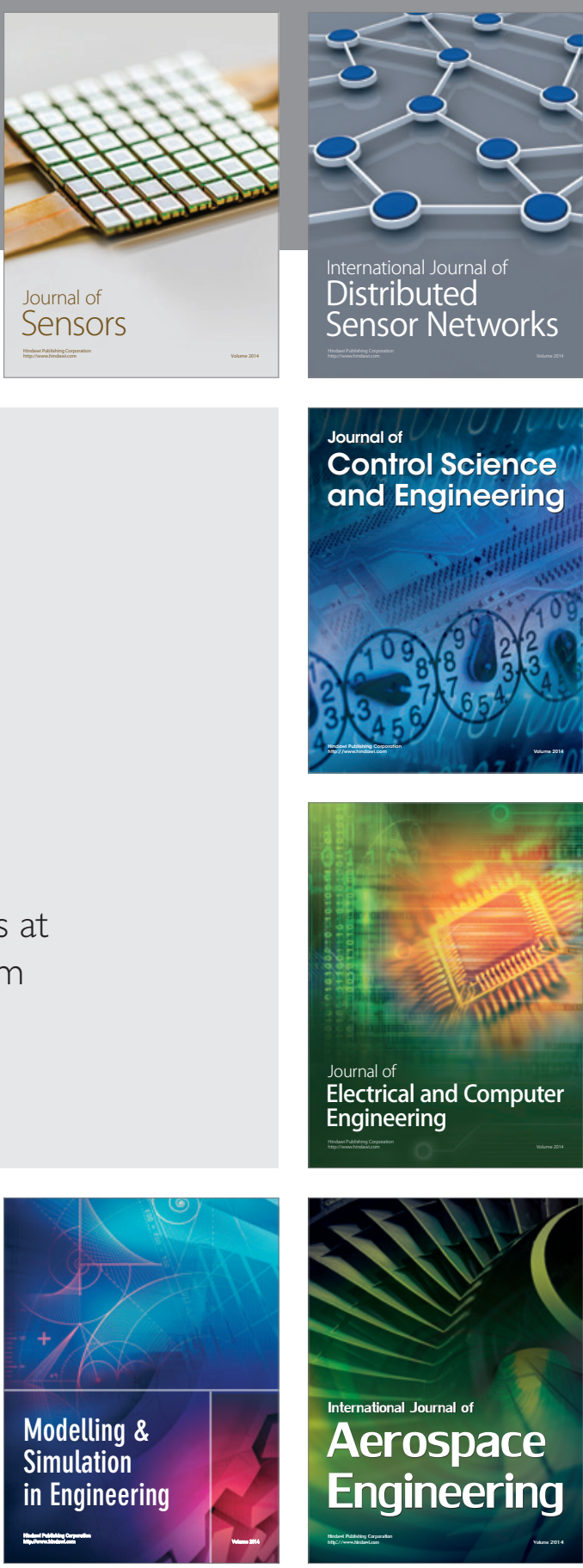

International Journal of

Distributed

Sensor Networks

Journal of

Control Science

and Engineering
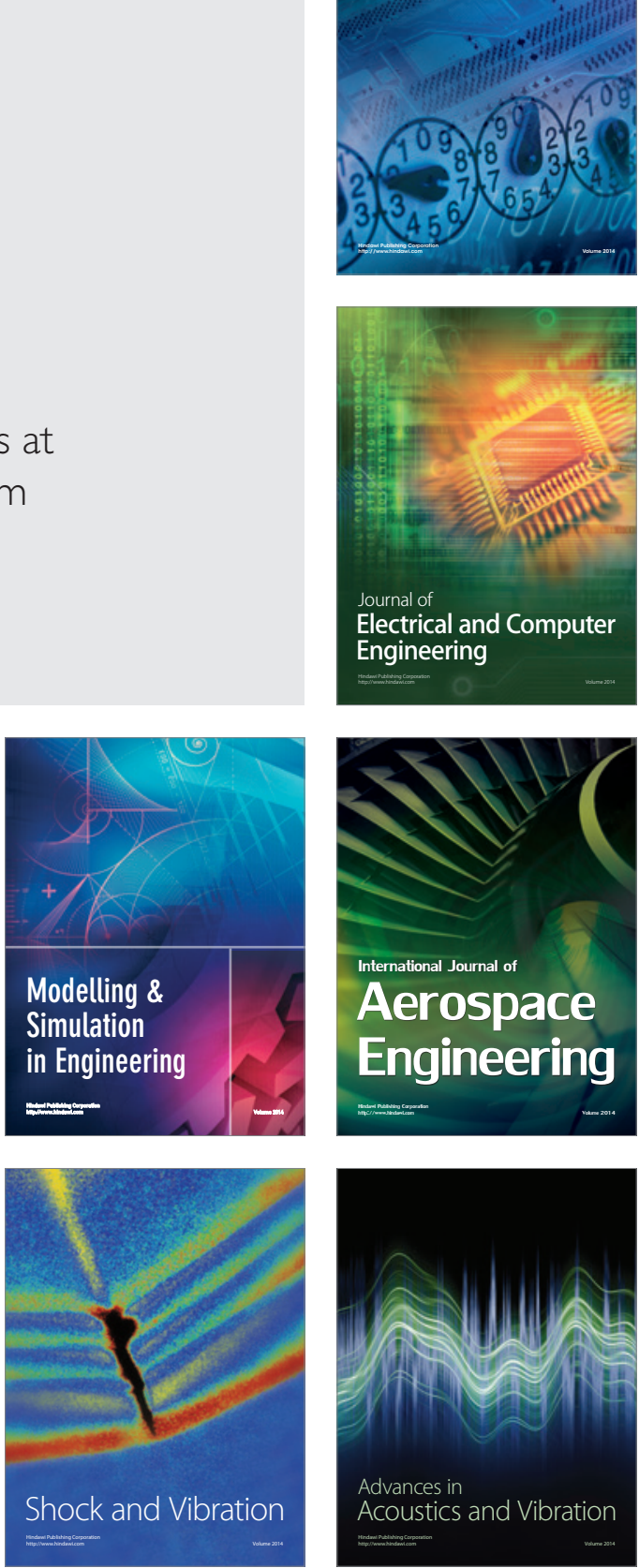